to chlorine and bromine was found to be practically $1: 6$, in the other five platinum alone was determined.

2. The successive crops of crystals obtained from a water solution of chloroplatinic acid and potassium bromide are not identical, but show gradually increasing proportions of the more soluble potassium platinibromide and correspondingly decreasing proportions of the less soluble potassium platinichloride.

3. On recrystallizing from water one of the substances under examination, the first crop of crystals is not identical with the original substance but is a product richer in chlorine and correspondingly poorer in bromine. This is to be expected on recrystallizing an isomorphous mixture of two substances differing in degree of solubility.

4. On treating one of the substances obtained with a quantity of water insufficient for complete solution, the more soluble bromide was dissolved from the mass to a greater extent than the less soluble chloride. This was confirmed both by the appearanch of the insoluble residue and its analysis.

At the beginning of this investigation Mr. Henry Hillyer, Jr., a student in this laboratory, was associated with me in the work. His sad death at his home in Augusta, Ga., on April 4, 1895, terminated this association. I desire to here pay tribute to his rare qualifications as an investigator and to the manliness which characterized his every act.

UNIVERSITY OF GEORGIA,

NoveMBER gth, I 895 .

\title{
THE QUALITATIVE EXAMINATION OF ACETANILID.
}

\section{By Charles Platt.}

Received November 22, 1895 .

I $N$ view of the deficiency in the analytical literature of the acid anilids the following tests may prove of interest. The formation of acetanilid, or phenylacetamid, $\mathrm{C}_{6} \mathrm{H}_{5} \mathrm{NHC}_{2} \mathrm{H}_{3} \mathrm{O}$, from anilin and glacial acetic acid

$$
\mathrm{C}_{6} \mathrm{H}_{5} \mathrm{NH}_{2}+\mathrm{C}_{2} \mathrm{H}_{3} \mathrm{O} . \mathrm{OH}=\mathrm{C}_{8} \mathrm{H}_{5} \mathrm{NHC}_{2} \mathrm{H}_{3} \mathrm{O}+\mathrm{H}_{3} \mathrm{O} \text {, }
$$

and the corresponding formation from anilin and acetyl chloride

$$
\mathrm{C}_{6} \mathrm{H}_{5} \mathrm{NH}_{2}+\mathrm{C}_{3} \mathrm{H}_{3} \mathrm{OCl}=\mathrm{C}_{6} \mathrm{H}_{5} \mathrm{NHC}_{2} \mathrm{H}_{3} \mathrm{O}+\mathrm{HCl} \text {, }
$$


suggest the adaptability of various color reactions with the acids and alkalies. The physical constants have been determined and the decomposition of the anilid made to serve for its identification.

Acetanilid is a white crystalline powder neutral in reaction and tasteless but producing a slight burning sensation when placed upon the tongue. Examined under the microscope it is found to be made up of broken crystalline plates. The melting point, commonly given as II $3^{\circ}-\operatorname{II} 4^{\circ}$, is determined by the writer as fairly constant at II $2^{\circ}$. Heated on platinum, acetanilid volatilizes without leaving a residue, and, when ignited, it burns completely with a yellow flame. At $15^{\circ}$ it is soluble in about I 90 parts of water and in five parts of alcohol. It is soluble in eighteen parts of boiling water and in about five-tenths part of boiling alcohol. It is soluble in the cold in ether, chloroform, acetic acid, nitric and sulphuric acids, and, when warmed, in hydrochloric acid. On boiling with water, oil-like globules separate out and the solution on cooling recrystallizes in large but delicate six-sided plates.

Reaction with Concentrated Nitric Acid.-Acetanilid is easily soluble in strong nitric acid, the solution being colorless in the cold but turning to a yellow, then brownish red, on gentle warming, nitrogen oxides being at the same time evolved. The colorless solution on standing in the cold gradually acquires a light green tint but finally changes, through yellow, to red, with formation of red acicular crystals. This red solution gives off the odor of nitrobenzene. A similar appearance and odor are produced by evaporating nitric acid solutions; an oily residue of decided odor being produced by rapid evaporation, a crystalline residue of slight odor by slow evaporation. The nitric acid solution has been described by one writer as colorless; by another as brown, then blue, then colorless.

Reaction with Dilute Nitric Acid.-The acetanilid is slowly soluble in the cold, and without change in color but with separation of oil-like globules. This solution on slow evaporation gives a brown residue with slight purplish tint. By boiling with dilute nitric acid a colorless solution is obtained, with evolution of pungent fumes. 
Reaction with Concentrated Sulphuric Acid.-A colorless solution is formed, unaffected by heating to boiling. The solution in cold concentrated acid, on long standing, acquires a pink to brown color. With an excess of the acetanilid the pink coloration develops quickly, is well marked, and changes gradually to an orange by reflected light. As a final change tufts of delicate acicular crystals appear and the solution gradually becomes colorless.

Reaction with Sulphuric Acid and Potassium Chromate.-A solution in concentrated sulphuric acid is turned to a dark green on addition of a few drops of potassium chromate. A solution in concentrated sulphuric acid, subsequently diluted, gives no reaction at first with the potassium chromate but on standing is gradually turned to a reddish-brown, and finally to a dark olive-green. A similar reaction to the last is produced by addition of the chromate to a solution in cold dilute acid.

Reaction with Hydrochloric Acid.-The acetanilid easily dissolves in hydrochloric acid when warmed and no precipitate is produced by subsequent dilution with water.

With hydrochloric acid and potassium dichromate there is no well marked reaction.

Reaction with Hydrochloric Acid and Potassium Permanganate.An olive-green coloration is obtained by adding a small crystal of the potassium permanganate to the solution in hydrochloric acid. On standing the color is changed to a mahogony-brown. Reaction with Hydrochloric Acid and Chromic Acid.-A solution of acetanilid in hydrochloric acid, diluted, and treated with a weak solution of chromic acid gives a yellowish-green coloration, which gradually turns to a dark green. Potassium hydroxide produces a blue precipitate in this solution.

Reaction with Hydrochloric Acid and Bromine.-Bromine water added in excess to a solution in hydrochloric acid produces a heavy yellow to white precipitate of the monobrom derivative of anilin. This precipitate examined microscopically is found to be made up of a fine interlacing net-work of needles or fiber-like crystals. A similar precipitate is obtained by means of bromine water in a water solution of the acetanilid.

Reaction with Hydrochloric Acid and Chlorine.-Chlorine water 
added to a solution in hydrochloric acid gives a dark blue coloration which afterwards fades. A similar reaction is obtained by substituting for the chlorine water a filtered solution of bleaching powder.

Mercuric chloride added to the hydrochloric acid solution gives no precipitate.

Dissolve some of the powder in a little hydrochloric acid and add, first, a few cc. of five per cent. phenol, then a little clear saturated solution of chlorinated soda or lime. The solution acquires a brownish-red color turning to a blue on addition of ammonium hydroxide in excess.

Reaction with Potassium Hydroxide.-By heating some of the powder with potassium or sodium hydroxide the characteristic odor of anilin is developed.

Reaction with Potassium Hydroxide and Chloroform.-By heating with potassium or sodium hydroxide and a few drops of chloroform the characteristic odor of an isonitrile is developed, phenylisocyanide being formed.

Reaction with Sodium Nitrite and Sulphuric Acid.-The powder mixed with sodium nitrate and sprinkled upon concentrated sulphuric acid produces a fine red coloration.

Reaction with Ferric Chloride.-A cold saturated water solution added to neutral ferric chloride produces no change in color.

Reaction with Zine Chloride.-Acetanilid heated to $270^{\circ}$ with an equal weight of zinc chloride produces, first, orthoamidoacetophenone, in small amount, and then flavanilin $\mathrm{C}_{6} \mathrm{H}_{14} \mathrm{~N}_{2}$, a yellow substance with a green fluorescence, a derivative of quinolin. It is stated in a number of text-books that acetanilid boiled with zinc chloride, anilin, and acetic acid will produce amidoacetophenone, $\mathrm{C}_{6} \mathrm{H}_{4} \mathrm{NH}_{2} \mathrm{C}_{2} \mathrm{H}_{3} \mathrm{O}$, but this test is untrustworthy inasmuch as the reagents used will produce this substance in absence of acetanilid. The paramidoacetophenone produced is crystalline in nature, while the ortho derivative formed in the previous test by heating zinc chloride and acetanilid is a yellow oil of high boiling point and with a characteristic sweetish odor.

Reaction with Plugge's Reagent.-Boil the acetanilid with water, cool and filter off if necessary, then boil again with potas- 
sium nitrite and dilute nitric acid. Mix with Plugge's reagent, a solution of mercurous nitrate with a little nitrous acid, and again heat to boiling. A deep red color is developed.

Antipyrin and phenacetin, two other popular antipyretics much used in medicine, may be readily distinguished from acetanilid by the foregoing tests. Antipyrin, for instance, with ferric chloride gives a deep-red coloration and is precipitated from its solutions by mercuric chloride. It has approximately the same melting point as acetanilid, but, unlike the latter, is decomposed by further heating. The characteristic reactions for phenacetin have been given by the writer in a former article.'

Comparative tests have been made upon various samples of acetanilid of domestic and foreign manufacture. The products of reputable houses seem to be practically identical with the exception of difference in perfection of crystallization and a corresponding difference in appearance.

CHEMICAL IABORATORY, HAHNEMANN

Meitcal College, Philadelphia.

\section{HEATS OF SOLUTION OF SOME CARBON COMPOUNDS.}

By C. L. SPEYERS.

Received October I4, 2895.

$T \mathrm{HE}$ following paper contains a few data about the heats of solution of some solid carbon compounds in water, methyl alcohol, ethyl alcohol, propyl alcohol, chloroform, and toluene.

The simple method given by Nernst ${ }^{2}$ was used.

The calorimeter was made of thin glass. The rim was ground to take the cover which fitted fairly well against the inside ground rim of the beaker, not air tight but tight enough to prevent appreciable evaporation of the more volatile solvents during the experiment. The cover had three holes with short tubulures. The center tubulure carried a test tube firmly fastened with a cork, the second tubulure as close to the center one as possible, carried the thermometer, while the third one, somewhat farther from the center, let the handle of the platinum stirrer pass through; see figure. The calorimeter held com-

1 J. Anal. Appl. Chem., 7, 2.

2 Ztschr.phys. Chem., 2, 23. 